\title{
On the equivalent flexural rigidity of sandwich composite panels
}

\author{
Jasson Gryzagoridis ${ }^{1}$, Graeme Oliver $^{1}$, Dirk Findeis ${ }^{2}$ \\ Mechanical Engineering Department \\ ${ }^{1}$ Cape Peninsula University of Technology \\ ${ }^{2}$ University of Cape Town \\ Cape Town - South Africa \\ E- Mail: gryzagoridisj@cput.ac.za
}

\begin{abstract}
The very graphic name of 'sandwich composites' adequately describes them as structures with a relatively thick core made of lightweight or low density material separating two thin stiff and strong skins. Such choice of geometry and combination of materials yields a product with reasonable strength and bending stiffness in combination with lightness. This paper presents work in predicting the bending stiffness of a sandwich composite through its equivalent flexural rigidity by modelling the material in the geometry of a cantilever beam. The results are verified experimentally by obtaining, through the laser based optical NDE technique known as Electronic Speckle Pattern Interferometry (ESPI), the displacement curve of the cantilever beam subjected to a point load at its free end. A second experimental technique carried out involved monitoring the dynamic response of a cantilever beam in its first mode of natural vibration. The beam equipped with polyvinyldiene fluoride (PVDF) sensors yielded results which are compared to the values for the flexural stiffness obtained by the prediction and the experimental setup using ESPI.
\end{abstract}

\section{Introduction}

The prime concern of designers being to improve the overall performance of systems has spearheaded advances in structures and new materials. Materials consisting of two or more different materials combined so that the resultant has more useful and meaningful applications than any of its individual components are constantly being created. These new materials are known as composites and have influenced just about every form of human endeavour. The prediction and/or measurement of a mechanical property identified as the flexural or bending stiffness of a particular type of composite material known as the sandwich structure is the subject of this paper. As the name implies the sandwich composite structure consists of two thin fairly strong and rigid faces or skins separated by a much thicker layer of lightweight and flexible material commonly referred to as core. The skins are normally adhesively bound to the core yielding a structure that has distinct advantages such as high bending stiffness to weight ratio, resistance to fatigue, good thermal insulation and damping characteristics, just to name a few.

Failure modes in sandwich structures are basically due to the nature of load applied and they are generally attributed to having exceeded the stiffness modulus of the composite resulting in failures such as local or general flexural crushing of core, tensile failure or wrinkling of the face etc. 


\section{Design considerations}

A sandwich structure is fundamentally designed to ensure that it possesses sufficient shear and flexural rigidity respectively to prevent failures as a result of large deflections due to excessive applied loads. When dealing with a sandwich composite beam its stiffness can be predicted on the basis of an equivalent flexural (EI) eq. rigidity or stiffness (the product of the material's Young's modulus and the moments of inertia) arising from the disparity of the skin and core Young's moduli and the geometry between core and skins. ${ }^{(1,2)}$ Refer to the dimensions of a beam as presented in figure 1: were $t$ is the thickness of the skins; $c$ is the thickness of the core, overall thickness of the sandwich is $d=c+2 t$, and $b$ and $l$ are the width and length of the specimen.

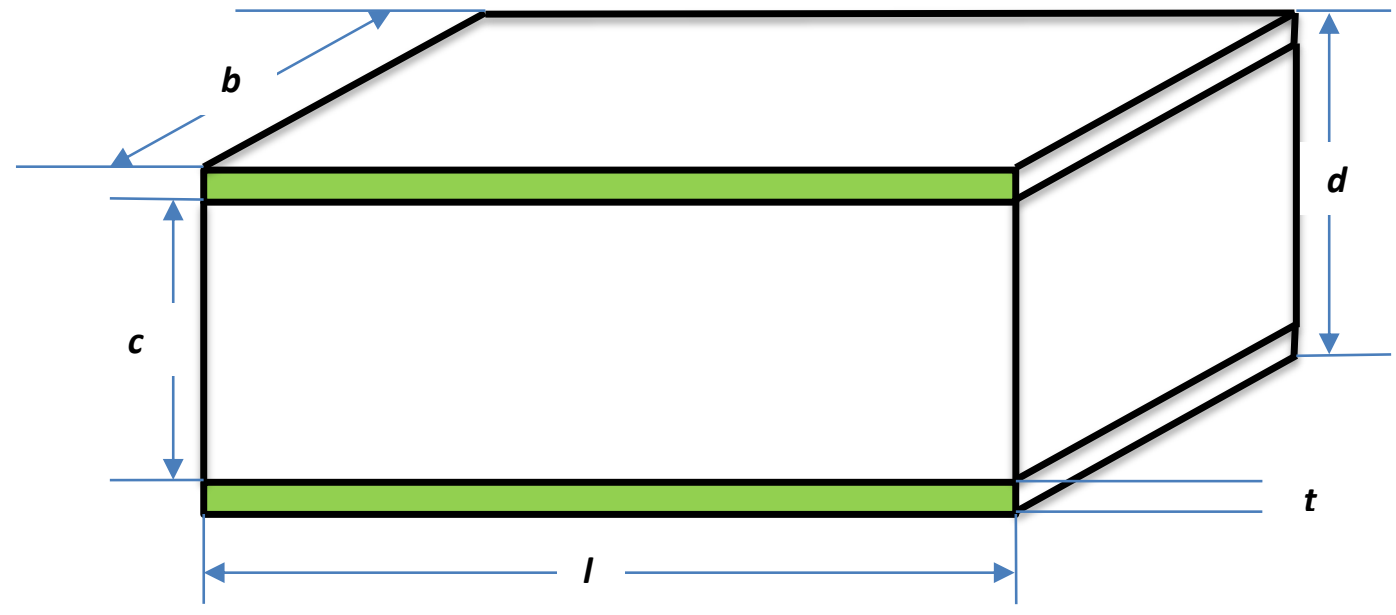

Fig.1 Schematic of a typical sandwich composite structure

Consider a cantilever beam made of sandwich geometry as shown in fig. 1 subjected to a load at its free end. The equivalent bending stiffness (EI) eq. can be represented as the sum of the bending stiffness of the core and of the two faces:

$$
(E I)_{e q .}=E_{c} I_{c}+2 E_{f} I_{f}
$$

Introducing the moments of inertial for the core and skins respectively and using the parallel axis theorem the outcome is,

$$
(E I)_{e q .}=\left(E_{c} b c^{3}\right) / 12+E_{f}\left[\left(b t^{3}\right) / 6+\left(b t d^{2}\right) / 2\right] .
$$

where $d$ is the thickness of the core and the two skins i.e. $c+2 t$. If one assumes that the skin's thickness $t$ is much thinner than the thickness of the core $c$ and that the Young's modulus of the core is at least an order of magnitude smaller than that of the skins, equation 2 can be simplified as a reasonable approximation:

$$
(E I)_{e q .}=E_{f}\left(b t d^{2}\right) / 2
$$


The total displacement of the end of a cantilever beam can be approximated by the bending deflection alone as the shear strength of the core should be high enough to prevent failure of the core and hence the shear component contributes negligibly to the total displacement which can be evaluated by the following classical cantilever beam's expression,

$$
\delta=\left(P L^{3}\right) /\left(3(E I)_{e q}\right)
$$

where $\delta$ is the total displacement of the free end, $\mathrm{P}$ is the load applied and $\mathrm{L}$ is the length of the beam.

\section{Experimental protocol}

Using equation 3 the bending stiffness (EI) eq. of a sandwich composite panel can be predicted provided of course that an accurate value of the Young's modulus of the skins/faces of the panel is known. This was accomplished by performing tensile tests on the material that was used as skins or faces when manufacturing the sandwich panels. The tensile tests of six skins yielded an average value for the Young's modulus of $6.2 \mathrm{GPa}$. Three different core material sandwich panels were manufactured with identical $1.49 \mathrm{~mm}$ (avg.) thick E-glass skins. Table 1 gives the dimensions and the different materials used in the manufacture of the sandwich composite panels and their predicted bending stiffness values according to equation 3.

Table1. - Sandwich Panel composition, dimensions \& (EI) eq.

\begin{tabular}{|c|c|c|c|c|c|}
\hline Item & Core & $\begin{array}{c}\text { Core } \\
\text { thickness }\end{array}$ & skin & $\begin{array}{c}\text { Skin } \\
\text { thickness }\end{array}$ & $\begin{array}{c}\text { (EI) eq. } \\
\mathrm{N} \mathrm{m}^{2}\end{array}$ \\
\hline 1 & Balsa Wood & $8.56 \mathrm{~mm}$ & E-glass & $1.49 \mathrm{~mm}$ & 25.2 \\
\hline 2 & Plastic & $8.95 \mathrm{~mm}$ & E-glass & $1.49 \mathrm{~mm}$ & 26.9 \\
\hline 3 & Foam & $10.52 \mathrm{~mm}$ & E-glass & $1.49 \mathrm{~mm}$ & 34.5 \\
\hline
\end{tabular}

The predicted values of the bending stiffness of the sandwich panels were subsequently validated by performing firstly, two simple experiments of measuring the free end deflection of cantilever beams and secondly, by determining the natural frequency of the $1^{\text {st }}$ mode of vibration of the same beams, which were fashioned from the sandwich composite panels shown in table 1 .

\subsection{Flexural stiffness obtained using a cantilever}

The experimental set up for the measurement of the deflection of the free end of a cantilever beam of known dimensions under a point load is very simple, requiring only rudimentary equipment such as a dial gauge, a few balance masses, a support, clamp etc. For a given point load or mass hung from the end of the cantilever beam a respective deflection will occur which should be recorded. Using the recorded deflection value in equation 4 the (EI) eq. can be solved for and compared to the one predicted from equation 3 .

The experimental apparatus consisted of the rig to grip the cantilever beam and the pulley arrangement to apply the load (masses) as depicted in fig. 2. The deflection of the beam was obtained using the author's proprietary portable Digital Shearography system ${ }^{(3)}$ which is transformable to Electronic Speckle Pattern Interferometer (ESPI) (see typical interferograms of 
the normal displacement of the beams in fig. 2). The normal displacement of the end point of the beam was calculated (for a load equivalent to 2.0 grams mass) using the expression:

$$
\delta=(N \lambda) /(\cos \alpha+\cos \beta)
$$

where $\delta$ is the normal displacement of a point in the surface of the beam associated (for simplicity) with a given number $(\mathrm{N})$ of the fringes exhibited on the ESPI interferograms; $\alpha$ and $\beta$ are the angles of illumination and observation to the beam's surface normal (in this case both approximated as zero) and $\lambda$ is the wavelength of the green laser used (532 nanometres). The choice to use the ESPI technique afforded minute loading and enhanced sensitivity.

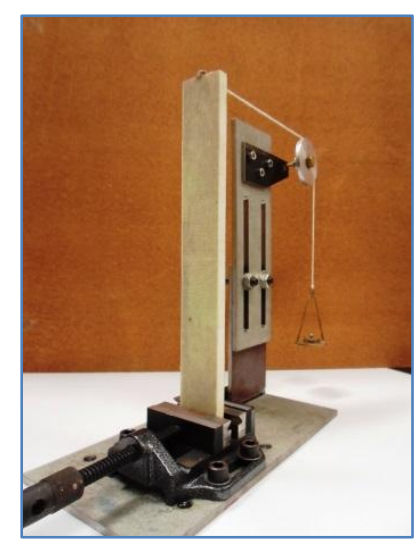

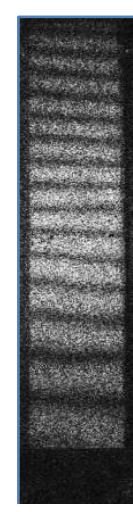

Balsa

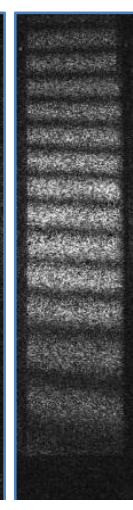

Plastic Foam

Fig. 2 Set-up for the measurement of the deflection of the free end of a cantilever and interferograms obtained using the ESPI technique

The second experiment of measuring the free end deflection of the cantilever beam was easily performed using a dial gauge to measure the beam's free end displacement that resulted from the loading of the beam as illustrated in figure 3 .

Table 2. - Cantilever dial gauge exp. data

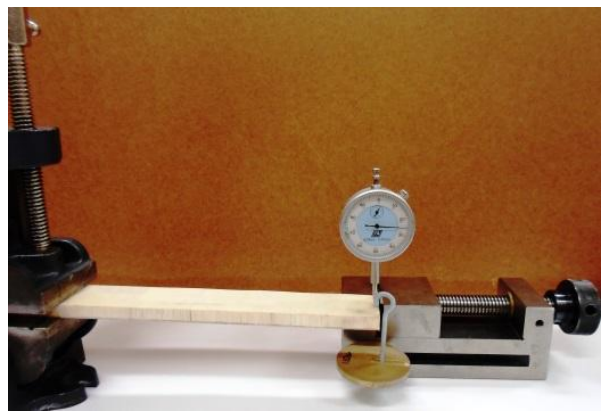

\begin{tabular}{|l|c|c|c|}
\hline $\begin{array}{c}\text { Core } \\
\text { Material }\end{array}$ & $\begin{array}{c}\text { Beam } \\
\text { length }\end{array}$ & $\begin{array}{c}\text { Load } \\
\text { grams }\end{array}$ & $\begin{array}{c}\text { Deflection } \\
\text { mm }\end{array}$ \\
\hline Balsa & $0.247 \mathrm{~m}$ & 110 & 0.225 \\
\hline Plastic & $0.247 \mathrm{~m}$ & 110 & 0.215 \\
\hline Foam & $0.247 \mathrm{~m}$ & 110 & 0.160 \\
\hline
\end{tabular}

Fig. 3 The measurement of the deflection of the cantilever's end using a dial gauge. 


\subsection{Flexural stiffness obtained via $1^{\text {st }}$ mode natural frequency of vibration.}

The objective of the experiments in determining the $1^{\text {st }}$ mode natural frequency of the various sandwich composite beams was to solve for their equivalent bending stiffness (EI) eq. $\left(\mathrm{Nm}^{2}\right)$ as these two quantities are related in the following expression ${ }^{(4,5,6)}$

$$
\Omega_{1}=C_{1}\left[(E I)_{e q} \cdot / \mu L^{4}\right]^{1 / 2}
$$

Where $\Omega_{1}$ is the natural frequency of mode one in $\mathrm{Hz}$. (cycles $/ \mathrm{sec}$ ); $\mathrm{C}_{1}=0.56$ is the constant for the first mode (with $\mathrm{C}_{2}=0.998$ and $\mathrm{C}_{3}=9.78$ for the second and third modes respectively, etc. just for information), $\boldsymbol{\mu}$ is the mass per unit length of the beam $(\mathrm{kg} / \mathrm{m})$ and $\mathrm{L}(\mathrm{m})$ is the effective length of the beam.

This experiment was designed on the basis of information from previously published work ${ }^{(7)}$ using Polyvinyldiene fluoride (PVDF) film, found to be very effective lightweight, durable and inexpensive sensor material. The film being flexible and lightweight when bonded to the surface of the beam will behave like "a dynamic strain gauge" (8) and will not affect the structure's response under dynamic conditions. The sandwich composite cantilever beam when deflected by laterally pushing on the free end and suddenly releasing it will begin to oscillate. The electrical charge that is generated by the flexing PVDF film sensor, because of its piezoelectric property, when fed into an oscilloscope will provide direct read-out of the cantilever's free vibration frequency. The experiments were very quick to perform (approximately 15 minutes to set up and obtain the average of 5 frequency readings for each beam) with the experimental set-up as depicted in fig. 3 below.

Table 3. - Cantilever's frequency exp. data

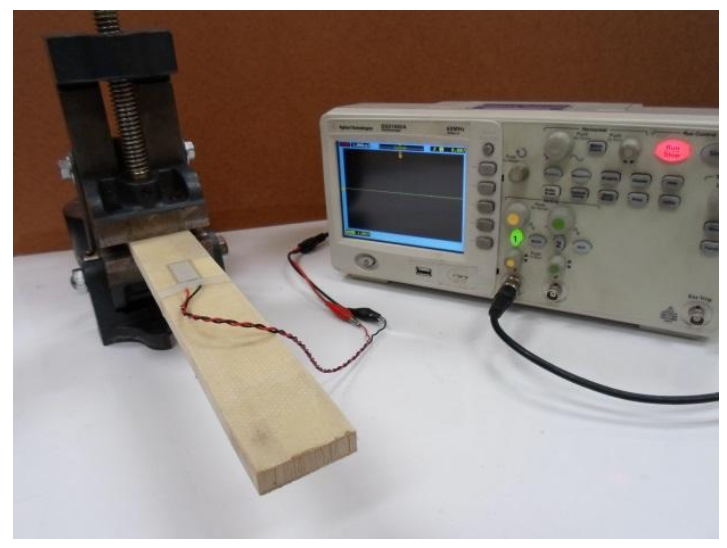

\begin{tabular}{|l|c|c|c|}
\hline $\begin{array}{c}\text { Core } \\
\text { Material }\end{array}$ & $\begin{array}{c}\text { Length of } \\
\text { cantilever } \\
\text { metres }\end{array}$ & $\begin{array}{c}\text { Mass/unit } \\
\text { length }-\mu \\
\mathrm{kg} / \mathrm{m}\end{array}$ & $\begin{array}{c}\text { Natural } \\
\text { frequency } \\
\mathrm{Hz} .\end{array}$ \\
\hline Balsa & 0.254 & 0.1853 & 99.4 \\
\hline Plastic & 0.254 & 0.1782 & 103.2 \\
\hline Foam & 0.254 & 0.1796 & 120.5 \\
\hline
\end{tabular}

Fig. 3 Experimental set-up to obtain the first mode natural frequency of the beam

\section{Summary of the results and conclusions}

In this paper the authors present results pertaining to the prediction of the equivalent flexural stiffness (EI) eq. of sandwich type composite panels and attempts to validate experimentally these predicted values. The predicted values (through equation 3) are based on assumptions regarding the disregard or omission of various parameters (presented in equation 2) which influence the behaviour of such composite panels when subjected to external loads. The resulting simplified 
mathematical expression appears to have the support of researchers as is evidenced in the literature ${ }^{(1,2,9)}$.

Three separate experimental approaches were employed to measure the equivalent flexural stiffness of the composite. The sandwich composite panel was modelled as a cantilever beam and the deflection of the end of the beam was measured firstly using a dial gauge, when a load was applied at the free end of the cantilever. A similar experiment was performed for cantilever beams that stood vertically and were loaded laterally through a pulley arrangement. The deflection of the beam, subsequent to applying the minute load from a mass of two grams, was obtained using the ESPI technique, well known for its high sensitivity in measuring extremely small displacements. Finally the frequency of the first mode of natural vibration of a cantilever was obtained through the use of a PVDF sensor attached on the cantilever beam.

The results of the experimental investigations in general compare well with the predicted values of the flexural stiffness, as it can be observed in table 2, where the maximum discrepancy is of the order of $5 \%$.

The results substantiate the claim that equation 3 is adequate to predict the flexural stiffness of sandwich type composite panels. To that end of course the Young's modulus of the skin material must be known, which is normally obtained through specimens stretched in a tensile testing machine. However a very simple, quick and inexpensive test using a dial gauge and some weights would suffice to establish the material's bending stiffness, without the need to determine the skins' Young's modulus.

Table 4.- Predicted and experimentally obtained flexural stiffness

\begin{tabular}{|c|l|c|}
\hline \multirow{4}{*}{ Core Material } & \multicolumn{1}{|c|}{ Methodology } & $\begin{array}{c}\text { (EI) eq. } \\
\mathrm{Nm}^{2}\end{array}$ \\
\hline \multirow{4}{*}{ Balsa Wood } & predicted from eq. 3 & 25.2 \\
\cline { 2 - 3 } & obtained through ESPI & 24.9 \\
\cline { 2 - 3 } & using a dial gauge & 24.1 \\
\cline { 2 - 3 } & from natural frequency & 24.3 \\
\hline \multirow{4}{*}{ Expanded Plastic } & 26.7 \\
\cline { 2 - 3 } & predicted from eq. 3 & 26.2 \\
\cline { 2 - 3 } & obtained through ESPI & 25.2 \\
\cline { 2 - 3 } & using a dial gauge & 25.2 \\
\cline { 2 - 3 } & from natural frequency & 34.5 \\
\hline \multirow{4}{*}{ Foam } & predicted from eq. 3 & 31.4 \\
\cline { 2 - 3 } & obtained through ESPI & 33.9 \\
\cline { 2 - 3 } & using a dial gauge & 34.6 \\
\cline { 2 - 3 } & from natural frequency & \\
\hline
\end{tabular}

\section{Acknowledgements}

The authors wish to express their appreciation to Armscor (The Armaments Corporation of South Africa) for the funding, through project Caliper with the University of Cape Town and in collaboration with Cape Peninsula University of Technology, which made this study possible. 


\section{References}

1. Xiang Li \& Gangyan Li \& Chun H. Wang (2012) “Optimization of Composite Sandwich Structures Subjected to Combined Torsion and Bending Stiffness Requirements" Appl. Compos Mater 19:689-704.

2. J. C. M. Theulen, A. A. J. M. Peijs ( 1991 ) “Optimization of the Bending Stiffness and Strength of Composite Sandwich Panels” Composite Structures 17 87-92.

3. J. Gryzagoridis, D. Findeis (2008) "ESPI of aerospace composites using digital Shearography Int. Symposium on NDT in Aerospace - Furth, Germany. Avail. From www.ndt.uct.ac.za last accessed June 2014.

4. P. J. Inman "Engineering vibration” Englewood Cliffs. NJ: Prentice Hall 1994.

5. J. Gryzagoridis, G. Oliver \& D. Findeis (2013) "Modal frequency vs. Shearography in detecting and locating voids/de-laminations in sandwich composites" Insight-Non Destructive Testing and Condition Monitoring, Vol. 55, No 5, pp. 249-252.

6. T. Irvine (2012) "Bending frequencies of Beams, Rods, and Pipes" www.vibrationdata.com/tutorials2/beam.pdf last accessed June 2014.

7. K. S. C. Kuang, S. T. Quek \& W. J. Cantwell (2004) "Use of polymer-based sensors for monitoring the static and dynamic response of a cantilever composite beam" Journal of Materials Science 393839 - 3843

8. Measurement Specialists Inc. "Piezo Film Sensors" Technical manual. www.meas-spec.com/ Last accessed June 2014.

9. A.C. Manalo, T. Aravinthan and W. Karunasena (2009) "Flexural behaviour of laminated fibre composite sandwich beams” proceedings APFIS 2009, pp. 407-412 